\title{
Diagnóstico de la enfermedad renal crónica como trazador de la capacidad técnica en la atención médica en 20 estados de México
}

\author{
Reyna Lizette Pacheco-Domínguez, M en C,(I) Luis Durán-Arenas, PhD(1), Mario Enrique Rojas-Russell, $M$ en $C^{(1,2)}$ \\ Ricardo A Escamilla-Santiago, MD, (') Malaquías López-Cervantes, MC, PhD.(I)
}

Pacheco-Domínguez RL, Durán-Arenas L, Rojas-Russell ME,
Escamilla-Santiago RA, López-Cervantes M.
Diagnóstico de la enfermedad renal crónica
como trazador de la capacidad técnica en la atención
médica en 20 estados de México.
Salud Publica Mex 20II;53 supl 4:S499-S505.

\section{Resumen}

Objetivo. Evaluar el conocimiento y la capacidad técnica de los médicos de primer nivel de atención en el manejo de los pacientes con diabetes mellitus e hipertensión arterial y de pacientes en riesgo de desarrollar enfermedad renal crónica (ERC) y utilizar la enfermedad terminal de esta última como trazador de la calidad de la atención primaria en el sistema de salud mexicano. Material y métodos. Se realizó un estudio transversal en los servicios de salud de las secretarías de salud en 20 estados de junio a diciembre de 2008. Se construyó un cuestionario con dos casos clínicos. Resultados. El promedio de calificación de los 149 médicos evaluados fue de 53.7 Los médicos que trabajan en las unidades de mayor tamaño tienden a tener mayor antigüedad y obtuvieron las calificaciones más bajas. Conclusión. La utilización del diagnóstico de la ERC como un trazador permite detectar la capacidad de los médicos en el primer nivel de atención y el potencial del uso de esta metodología para evaluar procesos críticos en el sistema de salud.

Palabras clave: trazadores; atención primaria de salud; ERC; conocimientos, actividades y prácticas en salud; México
Pacheco-Domínguez RL, Durán-Arenas L, Rojas-Russell ME, Escamilla-Santiago RA, López-Cervantes M.

The diagnosis of Chronic Kidney Disease as a tracer

of the technical capacity in care facilities

of 20 Mexican states.

Salud Publica Mex 20II;53 suppl 4:S499-S505.

\begin{abstract}
Objective. To assess knowledge and technical capacity of primary care physicians in the management of patients with diabetes mellitus and high blood pressure as well as patients at risk of developing chronic kidney disease, and to use the latter condition as a tracer of the quality of primary care of the Mexican health system. Material and Methods. A cross-sectional study included I 49 primary health physicians in primary care units from state health care services in 20 states. An instrument with two clinical cases was applied. Results. The average score of the physicians evaluated was 53.7 out of 100 . Those physicians working in larger size units and graduated before the year 2000 tend to receive lower scores. Conclusions. The use of chronic kidney disease as a tracer of the technical capacity of the Mexican health care system is useful to understand the problems of primary care in the country's public settings.
\end{abstract}

Key words: tracer method; primary health care; chronic kidney disease; health knowledge, attitudes, practice; Mexico

(I) Unidad de Proyectos Especiales de Investigación Sociomédica, Facultad de Medicina, Universidad Nacional Autónoma de México. México.

(2) Facultad de Estudios Superiores Zaragoza, Universidad Nacional Autónoma de México. México

Fecha de recibido: 30 de agosto de 20II - Fecha de aceptado: 2 I de septiembre de 201 I

Autor de correspondencia: Dr. Malaquías López-Cervantes. Unidad de Proyectos Especiales de Investigación Sociomédica, Facultad de Medicina, Universidad Nacional Autónoma de México. Edificio de Investigación, Ier. piso, Cd. Universitaria. 04510 México, DF, México.

Correo electrónico: mlopez@liceaga.facmed.unam.mx,mlopezl4@unam.mx 
$\mathrm{U}$ na evaluación no asegura calidad ni mejora la atención; sin embargo, es parte integral del desarrollo del sistema, un agente de cambio cuando el cambio es necesario y una herramienta para los educadores y consumidores al igual que para las fortalezas y debilidades del sistema. ${ }^{1}$

David Kessner y colaboradores propusieron el concepto de 'trazador' como una herramienta para evaluar la calidad de la atención médica. ${ }^{2}$ Es importante definir en nuestro idioma este concepto: por una parte, se le ha traducido como marcador, esto es, pensando en la sinonimia con una substancia que se detecta con facilidad y permite identificar procesos químicos, físicos o biológicos; por otra parte, se le puede definir como trazador, que es una sustancia que revela la existencia de un agente o proceso y que permite descubrir atributos sobre el agente o proceso, por ejemplo el carbono 14 utilizado en la datación de restos arqueológicos. Esta última definición es más cercana a lo que ofrece la metodología de referencia, es decir, permite que a través de un problema de salud (trazador) se identifiquen los atributos de calidad dentro de los servicios y sistemas de salud y de esta manera se obtiene evidencia del funcionamiento de todo el sistema.

Desde que se publicó el artículo original de Kessner a la fecha, han transcurrido casi 40 años; el proceso de transformación epidemiológica en el mundo continúa y México no es la excepción, así que el pool de enfermedades que propusieron como trazadores ha sido ampliado y las aplicaciones se han extendido. Por ejemplo, la Comisión Conjunta de Acreditación de Organizaciones de Salud utiliza la metodología de trazadores para su proceso de valoración de la calidad en las organizaciones sujetas al proceso de acreditación.

Sin embargo, en México no se ha hecho un uso amplio de esta metodología en los servicios de salud a pesar del reconocimiento internacional de la misma, como lo expresa Duncan Neuhauser en su revisión sobre este tema en $2004 .^{2}$

Por esta razón, ante la necesidad de estudiar la provisión de servicios de hemodiálisis en el sistema de salud mexicano, se decidió analizar la información disponible en nuestro estudio siguiendo la metodología de los trazadores; en este caso el trazador es la enfermedad renal crónica (ERC).

A nivel mundial, el número de pacientes con ERC se está incrementando marcadamente, especialmente en adultos, por lo que la ERC se ha reconocido actualmente como uno de los principales problemas de salud pública que amenaza con llegar a ser una epidemia de gran magnitud en la próxima década, ${ }^{3}$ por lo que sería adecuado que los profesionales de la salud tuvieran la capacidad para atender la demanda que ya lleva un tiempo expresándose.

Los datos de este estudio son parte de un trabajo mayor que elaboró un diagnóstico general de la necesidad y la demanda de atención médica que corresponden al síndrome de insuficiencia renal, así como de la situación que guardan los servicios de hemodiálisis en términos de su accesibilidad, utilización, efectividad y calidad, en las entidades federativas e instituciones de salud.

Un componente del trabajo fue evaluar la capacidad de las unidades de primer nivel de atención y las UNEME SoRID* para atender y dar seguimiento a los pacientes con diabetes mellitus e hipertensión arterial, así como para identificar a los pacientes en riesgo de desarrollar insuficiencia renal. Estas unidades son el enlace entre las unidades de primer nivel y las de segundo nivel de atención y cuentan con una red establecida de unidades de primer nivel a las que les deberían referir pacientes. ${ }^{4}$

Dadas las características de esta enfermedad y conforme con los criterios para que pueda ser un buen trazador de los servicios de salud, este padecimiento presentaba un reto metodológico de interés. Por una parte, en condiciones ideales su diagnóstico temprano y tratamiento oportuno deberían ser asequibles para el sistema de salud; sin embargo, se tenían evidencias de que el sistema de salud no estaba financieramente preparado para entregar los tratamientos sustitutivos que demanda este problema.

El propósito de este trabajo es evaluar el proceso diagnóstico que es financiado, por lo tanto, se entrevistó a los médicos que laboran en las unidades de primer nivel de atención en términos del conocimiento para atender y dar seguimiento a los pacientes con diabetes mellitus e hipertensión arterial, así como para identificar a los pacientes en riesgo de desarrollar insuficiencia renal, como trazador de la capacidad técnica del sistema de atención primaria.

\section{Material y métodos}

Se realizó un estudio transversal de investigación por encuesta en los servicios de salud de las secretarías de salud estatales en 20 estados. El estudio se realizó en los

\footnotetext{
* En 2007, la Secretaría de Salud de México creó una modalidad de unidad médica denominada UNEME SoRID (Sobrepeso, Riesgo Cardiovascular y Diabetes Mellitus) que tiene como objetivo contar con un modelo de atención institucional para atender a personas con padecimientos de sobrepeso, riesgo cardiovascular y diabetes mellitus, otorgándoles un manejo integral, interdisciplinario, basado en la evidencia científica, la experiencia clínica y las expectativas del paciente.
} 
meses de junio a diciembre de 2008 en 20 estados del país (ver sección de universo de estudio). Este estudio fue financiado por la Dirección de Evaluación del Desempeño de la Secretaría de Salud, por lo que a través de ésta se otorgó el consentimiento ético para realizar el estudio en todas las unidades médicas seleccionadas. Se aplicó una sección de consentimiento informado a cada médico cuando se le invitó a participar en la evaluación de manera anónima de forma tal que no afectara su integridad individual ni laboral.

\section{Universo de estudio y muestra}

Se seleccionó una muestra aleatoria de 95 unidades de primer nivel pertenecientes a la Secretaría de Salud en 20 entidades federativas (Aguascalientes, Campeche, Colima, Chihuahua, Durango, Guanajuato, Guerrero, Hidalgo, México, Morelos, Nayarit, Nuevo León, Oaxaca, Puebla, San Luis Potosí, Sinaloa, Tabasco, Tamaulipas, Veracruz y Zacatecas). El estudio es sólo generalizable al conjunto de los 20 estados y sus clínicas de primer nivel de las Secretarías de Salud Estatales y de la Secretaría de Salud Federal.

Dentro de estas unidades se entrevistó a todos los médicos en unidades de dos consultorios o menos, y se seleccionaron aleatoriamente dos médicos que atienden pacientes en las unidades que cuentan con más de dos médicos. Todos los médicos que fueron invitados aceptaron participar en el estudio.

\section{Instrumento}

Se construyó un cuestionario de opción múltiple que consta de 11 preguntas en donde se evalúa a los médicos en cuanto a su capacidad de diagnóstico y tratamiento de la diabetes mellitus, hipertensión arterial e insuficiencia renal crónica. Llevamos a cabo una validación que se realizó de tres formas: a) se elaboraron los casos clínicos que constituyen la base del instrumento a través de la metodología de elaboración de casos clínicos de la Universidad de Harvard; b) se realizó un piloteo con estudiantes de servicio social de la Facultad de Medicina a quienes se les aplicó el instrumento; y c) los resultados del piloto fueron revisados con un comité asesor constituido por nefrólogos.

El cuestionario consta de dos casos clínicos; en uno se presenta un paciente con diagnóstico incipiente de diabetes mellitus y se evalúa el manejo del paciente y el seguimiento para detectar ERC. En el segundo caso clínico se presenta un paciente con diagnóstico de hipertensión arterial no tratada y descontrolada y se evalúa el diagnóstico y manejo de la enfermedad, así como la detección de ERC (figura 1).

\section{Análisis}

Se construyeron diagramas de Kiviat para presentar el porcentaje de aciertos en cada ítem. Se realizaron comparaciones utilizando la prueba de $t, \chi^{2}$, o ANOVA de una vía, según fuera apropiado. El software que se utilizó para el análisis estadístico fue STATA, versión 10.

\section{Resultados}

En total se evaluaron 149 médicos egresados en su mayoría de escuelas de medicina públicas y que laboran principalmente en unidades de tipo urbano. Se encontraron médicos con años de titulación desde 1972 hasta 2009, y fueron predominantes los que se graduaron entre los años 2000 y 2007 (34\%). Cabe hacer mención que 11\% de éstos contaban con una residencia (37.5\% medicina familiar). De las 31 escuelas de medicina, en donde se formaron los médicos evaluados, a seis corresponde $52 \%$ de la población evaluada: Instituto Politécnico Nacional (10.3\%), la Benemérita Universidad Autónoma de Puebla (10.3\%), la Universidad Nacional Autónoma de México (9.6\%), la Universidad Veracruzana (8.1\%), la Universidad Autónoma Benito Juárez de Oaxaca (7.4\%) y la Universidad Autónoma de Sinaloa (6.7\%) (cuadro I).

Al evaluar el conocimiento de los médicos, de los 149 médicos a los que se aplicó el examen, se obtuvo un promedio de 53.7, con una calificación máxima de 90.1 y una mínima de 0 . El $64 \%$ obtuvo una calificación inferior a 6 .

Al analizar por año de graduación dividido en cinco grupos (1=1972 a 1979; 2= 1980 a 1989; 3= 1990 a 1999; $4=2000$ a 2007 y $5=2008$ a 2009 - pasantes) se observa una relación directamente proporcional entre la calificación y el año de graduación, que sin embargo no es estadísticamente significativa $(p=0.1072)$. Una situación similar se observó al ejecutar una prueba de tendencia con una correlación de 0.18 ( $p=0.065)$.

Cuando la diferencia se calculó entre dos generaciones (hasta 1999 y 2000 y más recientes) los médicos que se graduaron entre los años 2000 y 2009 obtuvieron calificaciones más altas en comparación con los que se graduaron entre los años de 1972 a 1999 (diferencia de promedios; $p=0.0133$ ). Es posible observar un patrón en el que los médicos que trabajan en las unidades de mayor tamaño tienden a ser graduados antes del año $2000(40 \%)$ y por lo tanto tendrían calificaciones más bajas; esto es contrario a las expectativas del estudio ya que se consideraba la experiencia o antigüedad como un aspecto positivo para el diagnóstico (figura 2).

No sólo la calificación global resultó baja, sino que al realizar un análisis de reactivos de los componentes 


\begin{tabular}{|c|c|c|c|}
\hline & Caso clínico I & & Caso clínico 2 \\
\hline $\begin{array}{l}\text { Mascl } \\
\text { mellit } \\
\text { hiper } \\
\text { mada } \\
\text { peso } \\
\text { explo } \\
\text { camp }\end{array}$ & $\begin{array}{l}\text { e } 55 \text { años de edad, con antecedentes familiares de diabetes } \\
\text { o } 2 \text { y cáncer de próstata por rama paterna, además de } \\
\text { narterial sistémica por línea materna; refiere que aproxii- } \\
\text { hace } 2 \text { meses inicia con poliuria, polidipsia y pérdida de } \\
\text { kg, motivo por lo cual acude hoy a consulta con usted. A la } \\
\text { fisica se encuentra paciente bien orientado, deshidratado, } \\
\text { nonares bien ventilados, área cardiaca con ruidos rítmicos, }\end{array}$ & $\begin{array}{l}\text { Masculino de } \\
\text { Tabaquismo } \\
\text { que padece } \\
\text { Desde hace } \\
\text { aumentado g } \\
\text { tipo punzant } \\
170 / 100 \mathrm{~mm}\end{array}$ & $\begin{array}{l}60 \text { años de edad, con antecedentes familiares de HAS e IAM por línea paterna. } \\
\text { positivo de } 40 \text { años a razón de } 30 \text { cigarrillos al día. Sedentario y obeso. Refiere } \\
\text { HAS desde hace cinco años, la cual no ha sido controlada adecuadamente. } \\
\text { un año ha presentado edema de miembros inferiores y fatiga, los cuales han } \\
\text { gradualmente. Refiere que desde hace dos semanas presenta dolor de cabeza } \\
\text { te que no cede con la ingesta de analgésicos por lo que acude a consulta. TA } \\
\text { hHg, FC 70x', FR } 19 \text { x', Peso } 122 \mathrm{~kg} \text {, Talla I.72 m, IMC } 41.23 \text {. }\end{array}$ \\
\hline $\mathrm{mmH}$ & 70x' Talla 1.70 m, Peso 9I kg, IMC 3I.487. & P2.1 & $\begin{array}{l}\text { Según las cifras de TA que presenta este paciente, ien qué estadio se encuen- } \\
\text { tra? }\end{array}$ \\
\hline PI.I & $\begin{array}{l}\text { ¿Qué estudios de laboratorio y/o gabinete solicita para } \\
\text { confirmar su diagnóstico? }\end{array}$ & P2.2 & $\begin{array}{l}\text { De las siguientes medidas terapéuticas para el manejo de la hipertensión, } \\
\text { ¿cuál NO está indicada en ese paciente? }\end{array}$ \\
\hline $\mathrm{PI} .2$ & $\begin{array}{l}\text { De los estudios anteriores ¿qué resultados espera } \\
\text { encontrar? }\end{array}$ & $\mathrm{P} 2.3$ & $\begin{array}{l}\text { ¿Qué estudios de laboratorio indicaría a este paciente, de acuerdo a su } \\
\text { padecimiento actual? }\end{array}$ \\
\hline $\mathrm{PI} .3$ & ¿Cluál es la actitud indicada ante este paciente? & & \\
\hline PI.4 & ¿Cuándo está indicada la próxiima consulta de este & Los estudios & s de laboratorio reportaron los siguientes resultados anormales: \\
\hline $\begin{array}{l}\text { Tras } \\
\text { con u } \\
\text { cifras } \\
\text { repor }\end{array}$ & $\begin{array}{l}\text { eses de la primera consulta acude el paciente nuevamente } \\
\text { refiere que cedió la sintomatología inicial, manteniendo } \\
\text { ccemia dentro de parámetros normales, sin embargo se } \\
\text { roalbuminuria de más de } 30 \mathrm{mg} / 24 \text { horas. }\end{array}$ & $\begin{array}{l}\text { QS: } \\
\text { Creatinina: } \\
\text { Urea: } \\
\text { BUN: } \\
\text { EGO: } \\
\text { P2.4 }\end{array}$ & $\begin{array}{l}\text { Glucosa: } 214 \mathrm{mg} / \mathrm{dl} \\
1.8 \mathrm{mg} / \mathrm{dl} \\
60 \mathrm{mg} / \mathrm{dl} \\
30 \mathrm{mg} / \mathrm{dl} \\
\text { Microalbuminuria mayor de } 30 \mathrm{mg} / 24 \text { horas } \\
\text { En este paciente ¿cómo se hace el diagnóstico de IRC? }\end{array}$ \\
\hline $\mathrm{PI} .5$ & $\begin{array}{l}\text { ¿Qué actitud toma con respecto a la evolución de este } \\
\text { paciente? }\end{array}$ & $\begin{array}{l}\mathrm{P} 2.5 \\
\mathrm{P} 2.6\end{array}$ & $\begin{array}{l}\text { Si el paciente se encontrara con GRF de } 40 \mathrm{ml} / \mathrm{min} \text {, ¿en qué etapa de insu- } \\
\text { ficiencia renal crónica se encuentra? } \\
\text { ¿Cuál es la complicación más frecuente de la insuficiencia renal crónica en } \\
\text { la fase terminal? }\end{array}$ \\
\hline $\begin{array}{l}\text { HAS }=1 \\
\text { IAM }=\text { i } \\
\text { IRC }=\text { in }\end{array}$ & $\begin{array}{l}\text { sión arterial sistémica } \\
\text { gudo del miocardio } \\
\text { ccia renal crónica }\end{array}$ & & \\
\hline
\end{tabular}

Figura I. Cuestionario de evaluación de personal médico sobre capacidad de diagnóstico y tratamiento de la

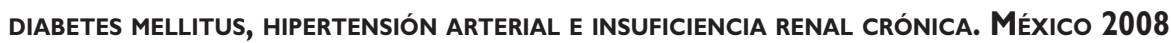

\section{Cuadro I}

Características de los médicos. Unidades de primer nivel Pertenecientes a la Secretaría de Salud en 20 entidades federativas. México 2008

\begin{tabular}{|c|c|}
\hline Características & No. (\%) \\
\hline \multicolumn{2}{|l|}{ Tipo de escuela } \\
\hline Pública & $136(94)$ \\
\hline Privada & $9 \quad(6)$ \\
\hline \multicolumn{2}{|l|}{ Tipo de unidad } \\
\hline Rural & $14(9)$ \\
\hline Urbana & $135(9 \mid)$ \\
\hline \multicolumn{2}{|l|}{ Año de titulación } \\
\hline $1972-1979$ & II (8) \\
\hline $1980-1989$ & $35(26)$ \\
\hline $1990-1999$ & $38(28)$ \\
\hline $2000-2007$ & $46(34)$ \\
\hline 2008-2009 (pasantes) & $5 \quad(4)$ \\
\hline \multicolumn{2}{|l|}{ Tamaño de la unidad } \\
\hline$<3$ núcleos básicos & $33(22)$ \\
\hline$\geq 3<7$ núcleos básicos & $5 I(34)$ \\
\hline$\geq 7$ núcleos básicos & $65(44)$ \\
\hline Médicos con residencia & $16(11)$ \\
\hline
\end{tabular}

del proceso de atención se pudo observar que después de haber obtenido el mayor porcentaje de respuestas correctas en preguntas sobre el manejo y los estudios diagnósticos de laboratorio que deben llevarse a cabo, $70 \%$ de los médicos interpretaron incorrectamente los resultados (figura 3).

La calificación de los médicos no es modificada por la ubicación de la unidad (rural o urbana), por la escuela de procedencia, ni por si realizaron una residencia o no.

\section{Discusión}

El proceso de la atención médica está bajo el control del médico y en consecuencia, la responsabilidad finalmente radica en él. Sin embargo, cuando se encuentra evidencia que muestra que la atención no es tan buena como pudiera ser, ésta tiende a ser descalificada y a considerar los datos utilizados como erróneos o poco científicos, por lo tanto no se toman en cuenta y se ocultan. ${ }^{2}$ Esta es una de las grandes limitaciones de las evaluaciones de calidad que no se acompañan de un proceso de mejora continua de la misma.

Los estudios epidemiológicos que se han realizado han puesto en evidencia que la enfermedad renal terminal 
(a) Promedio del examen realizado por los médicos según año de grraduación y tamaño de la unidad

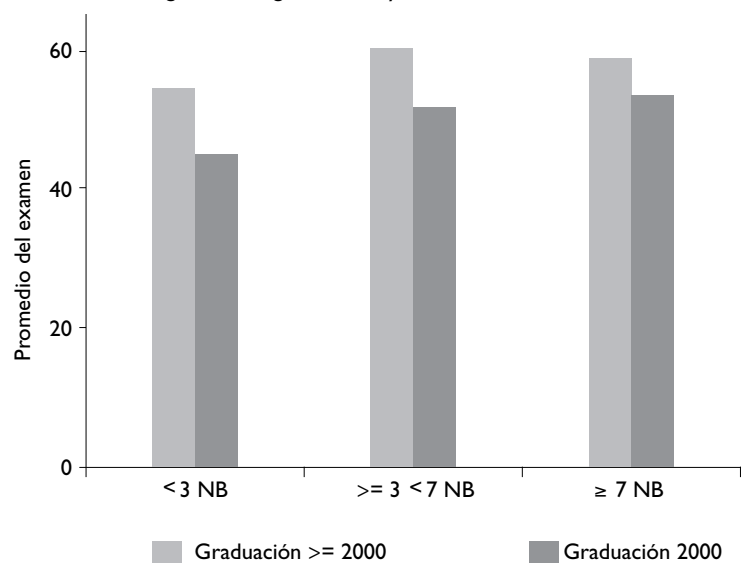

(b) Médicos según año de graduación y tamaño de la unidad

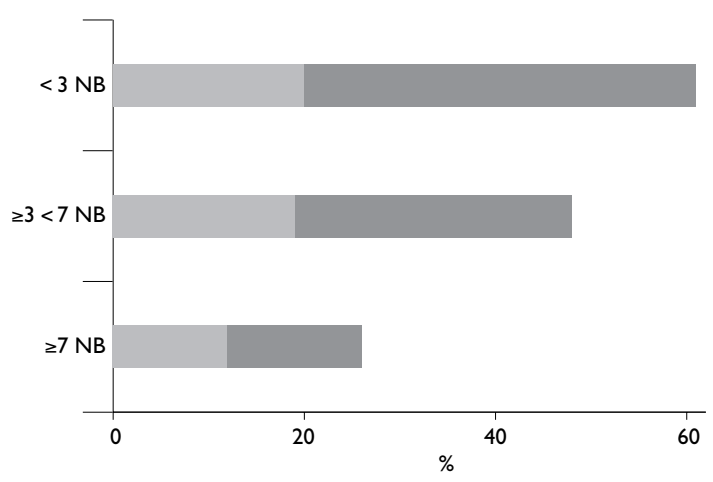

Graduación antes del año $2000 \quad$ Graduación en o desués del año 2000

Figura 2. Promedio de la evaluación en relación con el año de graduación y tamaño de la unidad. Unidades de primer nivel pertenecientes a la Secretaría de Salud en 20 entidades federativas. México 2008.

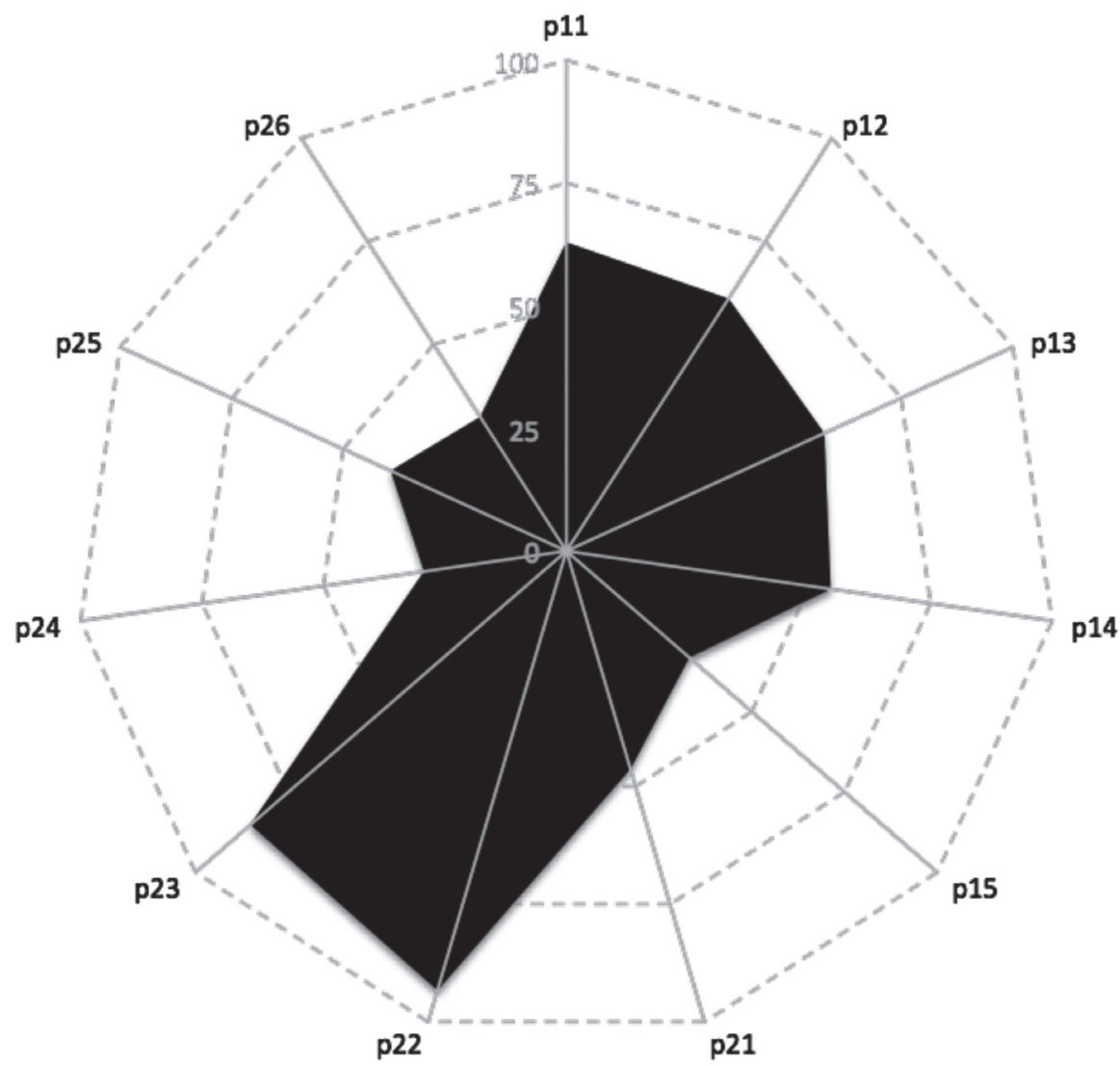

Figura 3. Porcentaje de aciertos de los médicos en cada ítem. Unidades de primer nivel pertenecientes a la Secretaría de Salud en 20 entidades federativas. México 2008 
(ERT) representa la punta del iceberg de la ERC y sugieren que aquellos pacientes en estadios tempranos exceden al menos 50 veces a aquellos con ERT. ${ }^{5}$ Las estadísticas de mortalidad en el año 2005, en México, mostraron que la ERT fue, por sí misma, la décima causa de muerte a nivel nacional; de acuerdo con datos disponibles en el país, Franco y cols. determinaron que existen actualmente 129 mil pacientes con ERT, ${ }^{*}$ por lo que se esperarían alrededor de 6.45 millones de personas con ERC en etapas tempranas. Si la capacidad diagnóstica es limitada, muchos de estos casos no serán detectados oportunamente.

La ERC no debe verse aisladamente ya que presenta una interacción muy compleja con la enfermedad cardiovascular, la diabetes mellitus y la hipertensión arterial. ${ }^{6}$ En México, la diabetes mellitus y la hipertensión arterial se han incrementado en los últimos años. En 2000 se reportó una prevalencia de diabetes mellitus de $7.2 \%{ }^{7}$ y en 2006 una prevalencia de hipertensión arterial de $16.3 \%,{ }^{8}$ y al ser ambas potenciales causas de ERC, se convierten en el blanco perfecto para prevenirla, pues se deben detectar en etapas tempranas y manejar adecuadamente no sólo a los pacientes con ERC sino también a aquellos pacientes con diabetes, hipertensión y enfermedad cardiovascular.

En el caso de este proyecto, se estudió la calidad del diagnóstico del médico debido a que la prevalencia de las enfermedades asociadas con la ERC está muy probablemente subestimada en el primer nivel de atención, a pesar de ser uno de los principales problemas de salud pública por el alto costo que implica la terapia de sustitución y por la clara y compleja interacción entre la ERC, la diabetes mellitus, la hipertensión y las enfermedades cardiovasculares. ${ }^{9}$ La evidencia sugiere que el potencial de subestimar por la baja calidad diagnóstica de los médicos no es despreciable.

En este contexto se debe considerar nuestro hallazgo más importante: "la mayoría de los médicos obtuvieron calificaciones bajas; los graduados en años anteriores al 2000 obtuvieron las calificaciones más bajas y tienden a trabajar en las unidades más grandes".

Es contradictorio que las unidades más grandes no cuenten con médicos actualizados; se esperaría que en estos lugares existieran mayores recursos de información. Por otra parte, es lógico esperar que la mayor necesidad de actualización sea en los que tienen muchos años de graduados, pero en general las calificaciones

\footnotetext{
* Franco-Marina F, Tirado-Gómez L, Venado-Estrada, A, Moreno-López JA, Pacheco-Domínguez RL, Durán-Arenas L, López-Cervantes M. Una estimación indirecta de las desigualdades actuales y futuras con respecto a la frecuencia de la enfermedad renal crónica terminal en México. (Enviado para publicación).
}

de los médicos son bajas en todos (6.7\% de los médicos tuvieron calificaciones por arriba de 80).

Esto nos genera una serie de preguntas para explicar esta situación: ¿son las condiciones de la atención de primer nivel en general tan adversas que no permiten una práctica médica óptima?; ¿existe falta de conocimientos en los médicos de atención primaria sobre la importancia de las medidas preventivas?; ¿ es la falta de cursos de educación continua la causa fundamental para la falta de actualización de los conocimientos de los médicos en ejercicio?; ${ }^{10}$ ¿existe falta de compromiso de la comunidad médica?; y por supuesto, ¿son los escasos recursos que se destinan a la medicina preventiva por parte del sistema nacional de salud la causa principal? ${ }^{11}$

En este sentido, Jorm y Kam sugieren un acercamiento sistemático para mantener los estándares del personal profesional y establecer como objetivo de la práctica médica la medicina basada en la evidencia; de acuerdo con estos autores, "mientras es raro encontrar malos doctores es muy común encontrar rústicos y arcaicos". Nuestros hallazgos coinciden con esta declaración. Y repitiendo su frase: " ¿Es posible que la cultura de Camelot sea esencial para la actual sobrevivencia de los caballeros? Tal vez hay un camino en que los caballeros se armen de valor para matar al dragón, pero si no existe una cultura, rituales o artefactos que delineen al caballero, no lo va a hacer bien", ${ }^{10}$ estos aspectos parecen faltar también en nuestro sistema de salud.

A pesar de que la evaluación que se les aplicó no puede concluir si el diagnóstico que se realizaría al estar frente a un paciente real pueda hacerse correctamente o no, nos sugiere que los médicos no cuentan con la capacidad y conocimiento que les permitan realizar su trabajo en el caso de la ERC. Es necesario determinar si las instituciones de salud no ofrecen a los recursos humanos actualización o los médicos no la buscan, o ambas, ya que se esperaría que los profesionales realicen un trabajo con altos estándares sin supervisión y siguiendo los principios éticos de la profesión. ${ }^{12}$

Es necesario también considerar otros aspectos que se pudieran asociar con esta situación, pues aunque se contara con los recursos adecuados para poder llevar a cabo la prevención de ERC nuestros hallazgos sugieren que aun así no sería posible realizarla porque quienes deberían llevarlas a cabo no tienen conocimiento de ella. En futuros estudios se buscaría identificar factores asociados con la estructura de las unidades, más allá de las calificaciones de los médicos.

Si bien el estudio reflejó un sesgo al tener en su muestra principalmente unidades de primer nivel urbanas y de mayor tamaño, el sesgo sería a favor de los hallazgos del estudio pues es muy probable que en 
las unidades más pequeñas y rurales los médicos se encuentren en condiciones de estructura y recursos más limitados, y por lo tanto no estén capacitados para poder diagnosticar pacientes con ERC y ni siquiera cuenten con los exámenes de laboratorio que son básicos para el diagnóstico.

Con base en los resultados obtenidos en el presente estudio podemos señalar que en México hay una deficiencia en la atención primaria de la salud, la cual desde hace más de un cuarto de siglo es reconocida como uno de los componentes claves de un sistema de salud efectivo. ${ }^{13} \mathrm{La}$ alta prevalencia de la ERC, la ausencia de síntomas hasta que la enfermedad llega a estadios avanzados, la accesibilidad a las pruebas de laboratorio para realizar el diagnóstico y el pronóstico, y la disponibilidad de los tratamientos para prevenir complicaciones sugieren que el tamizaje para ERC puede ser de gran valor. ${ }^{14}$

Tanto para los individuos como para la sociedad, resulta menos costoso y más efectivo cubrir los gastos médicos en un sistema de salud enfocado a la atención primaria de la salud, en comparación con sistemas de atención orientados hacia la atención especializada, como sucede en México.

Sin embargo, nuestros resultados nos muestran que es necesario no sólo destinar más recursos sino fortalecer la organización de la atención de primer nivel, ya que existe consenso de que la tarea principal de las autoridades en salud de los países en desarrollo es detectar y tratar las enfermedades en el estadio más temprano posible. ${ }^{15}$ Esto es particularmente importante en el caso de las enfermedades crónicas. El uso de la metodología de trazadores nos permitió identificar algunos de los aspectos asociados con los problemas del diagnóstico de la ERC. Si esta situación es un reflejo de las condiciones de la práctica médica en el primer nivel de atención del país, la utilización del diagnóstico de la ERC como un trazador para detectar la capacidad del sistema de salud de México nos deja con una gran preocupación por la transformación del sistema. No lograremos nuestros objetivos de salud únicamente con más dinero; es necesario transformar el sistema de salud a través del desarrollo de la atención primaria.
Declaración de conflicto de intereses: Los autores declararon no tener conflicto de intereses.

\section{Referencias}

I. Kessner DM, Kalk CE, Singer J. Assessing health quality--the case for tracers. N Engl J Med 1973; 288(4):189-194.

2. Neuhauser D. Assessing health quality: the case for tracers. J Health Serv Res Policy 2004; 9(4):246-247.

3. Lysaght MJ. Maintenance dialysis population dynamics: current trends and long-term implications. J Am Soc Nephrol 2002; I3 Suppl I:S37-S40. 4. Secretaria de salud. UNEMES Unidades de Especialidades médicas. México 2007. [Consultado el 25 de mayo de 2010]. Disponible en: http:// portal.salud.gob.mx/sites/salud/descargas/pdf/unemes07.pdf. 5. Coresh J, Astor BC, Greene T, Eknoyan G, Levey AS. Prevalence of chronic kidney disease and decreased kidney function in the adult US population: Third National Health and Nutrition Examination Survey. Am J Kidney Dis 2003; 4 I (I):I-I2.

6. Atkins RC. The changing patterns of chronic kidney disease: the need to develop strategies for prevention relevant to different regions and countries. Kidney Int Suppl 2005; (98):S83-S85.

7. Secretaría de Salud, Instituto Nacional de Salud Pública. Encuesta Nacional de Salud (ENSA 2000). México: SSA/INSP, 2000.

8. Olaiz-Fernández G, Rivera-Dommarco J, Shamah-Levy T, Rojas R, Villalpando-Hernández S, Hernández-Avila M, et al. Encuesta Nacional de Salud y Nutrición 2006. Cuernavaca, México: Instituto Nacional de Salud Pública, 2006.

9. Schieppati A, Remuzzi G. Chronic renal diseases as a public health problem: epidemiology, social, and economic implications. Kidney Int Suppl 2005; (98):S7-SIO.

10. Pearson P, Jones K. Primary care-opportunities and threats. Developing professional knowledge: making primary care education and research more relevant. BMJ 1997; 314(7083):817-820.

I I. Correa-Rotter R, González-Michaca L. Early detection and prevention of diabetic nephropathy: a challenge calling for mandatory action for Mexico and the developing world. Kidney Int Suppl 2005; (98):S69-S75.

12. Jorm C, Kam P. Does medical culture limit doctors' adoption of quality improvement? Lessons from Camelot. J Health Serv Res Policy 2004; 9(4):248-25I.

13. Cueto $M$. The origins of primary health care and selective primary health care. Am J Public Health 2004; 94(II): I864-1874.

14. James MT, Hemmelgarn BR, Tonelli M. Early recognition and prevention of chronic kidney disease. Lancet 2010; 375(9722): 1296-1309.

I5. Barsoum RS. Chronic kidney disease in the developing world. N Engl J Med 2006; 354(I0):997-999. 